\title{
Efficacy of the Inflammatory Cell Infiltration Inhibitor IS-741 on Colitis Induced by Dextran Sulfate Sodium in the Rat
}

\author{
Shuichi Yotsuya*, Hiroshi Shikama and Masashi Imamura \\ Central Research Institute, Ishihara Sangyo Kaisha, Ltd., 2-3-1, Nishi-Shibukawa, Kusatsu, Shiga 525-0025, Japan
}

Received March 1, 2001 Accepted August 3, 2001

\begin{abstract}
We examined the therapeutic effects of the inflammatory cell infiltration inhibitor IS-741 $(\mathrm{N}-$ (2-((ethylsulfonyl)amino)-5-(trifluoromethyl)-3-pyridinyl)-cyclohexanecarboxamide monosodium salt monohydrate) on a rat colitis model. As a result of its effects on leukocyte infiltration, IS-741 inhibits cell adhesion, alleviates symptoms and signs of pancreatitis and multiple organ failure and demonstrates a lifesaving effect in a model of severe acute pancreatitis. A rat model was prepared by inducing colitis with $3 \%$ dextran sodium sulfate (DSS) and maintaining pathology with 1\% DSS. Repeated oral administration of IS-741 at 1,10 or $100 \mathrm{mg} / \mathrm{kg}$ per day was conducted for 2 weeks (during treatment with $1 \%$ DSS). IS-741 at each dose decreased the area of erosion in the large intestine, thickening of the wall of the large intestine and anemia caused by melena. Some effects of IS-741 were nearly equivalent to those of the control compound salazosulfapyridine. Furthermore, IS-741 markedly alleviated inflammatory cell infiltration into the intestinal wall. IS-741 improved lesions in a rat DSS model by inhibiting leukocyte infiltration, suggesting the possibility of clinical application of this drug for IBD.
\end{abstract}

Keywords: IS-741, Inhibition of cell adhesion, Inhibition of leukocyte infiltration, Inflammatory bowel disease, Ulcerative colitis

Since the cause of inflammatory bowel disease (IBD) is still uncertain, methods for treatment of IBD aimed at elimination of its cause have yet to be established. In this regard, various methods of treatment of IBD have been investigated.

Metabolites of arachidonic acid are mediators that directly induce intestinal tract inflammation. The involvement of thromboxane $\mathrm{A}_{2}\left(\mathrm{TXA}_{2}\right)$ generated by cyclooxygenase and leukotriene $\mathrm{B}_{4}\left(\mathrm{LTB}_{4}\right)$ generated through lipoxygenase in pathogenesis of IBD has been reported (1). Increase in $\mathrm{LTB}_{4}$ production is known to be closely related to acute inflammation. In this regard, $\mathrm{LTB}_{4}$ is considered an important factor aggravating the inflammatory reaction (2). A relationship between $\mathrm{TXA}_{2}$ and persistent chronic inflammation has also been found. Salazosulfapyridine (SASP) and adrenocortical steroids, which are extensively used as effective therapeutic drugs for IBD, both inhibit the metabolic pathway of arachidonic acid, and inhibition of the generation of $\mathrm{TXA}_{2}$ and $\mathrm{LTB}_{4}$ is believed to be their mechanism of action.

Recently, the abnormal immunological condition caused

*Corresponding author. FAX: +81-77-562-9783

E-mail: s-yotsuya@iskweb.co.jp by IBD has been clarified to some extent, and it has been found to be closely related to the occurrence of IBD. Studies on the abnormal immunological condition in IBD are being conducted from various aspects, including increase in IgG-containing cells, precipitation of complement in the intestinal mucosa, abnormality of cellular immune mechanisms and cytokine function (anti-TNF $\alpha$ antibody or anti-IL-10 antibody $(3,4))$. Clinically, drugs with immunosuppressive effects are used in the treatment of $\operatorname{IBD}(5,6)$.

On the other hand, oxygen-free radicals produced by neutrophils and macrophages and nitrogen monoxide generated from vascular endothelium are also assumed to cause tissue damage $(7,8)$. In this connection, the study of superoxide dismutase is proceeding. Recently, the involvement of neutrophils in the pathogenesis of IBD is attracting attention, and treatments using fiber filters that remove only excessively activated neutrophils are being investigated. It is reported that this method is very effective in inducing remission of IBD that does not respond to the above-mentioned drug therapy and that such remission can be maintained by periodic removal of neutrophils $(9-12)$. In addition, the involvement of cell adhesion molecules in IBD is attracting particular attention (13-17), and a study 
has begun to inhibit neutrophil function with the use of drugs (eNIF: recombinant neutrophil inhibitory factor (18), ICAM-1 antisense (19)).

Onset of IBD is noted in comparatively young individuals. Despite the presence of various treatment methods, cure is difficult and patients suffer from this disease for a long period of time. Furthermore, in addition to the substantial physical and financial burden on patients, it is often difficult to continue treatment due to decreased therapeutic effects or adverse reactions $(20,21)$, over the course of time. In this regard, use of IS-741 may expand the choices available for treatment of IBD, simplifying prescription or therapeutic technique and decreasing the physical and financial burden on patients. IS-741 has been demonstrated to inhibit leukocyte infiltration and to alleviate various types of inflammation by inhibiting cell adhesion $(22-27)$. Since IS-741 is expected to be efficacious against IBD, in which leukocyte infiltration plays an important role in pathogenesis, the effect of IS-741 was investigated in a typical rat IBD model of ulcerative colitis induced by dextran sodium sulfate (28).

\section{MATERIALS AND METHODS}

\section{Test substance}

The test compound IS-741 ( $N$-(2-((ethylsulfonyl)amino)5-(trifluoromethyl)-3-pyridinyl)-cyclohexanecarboxamide monosodium salt monohydrate) (Fig. 1) is a diaminopyridine derivative created by Ishihara Sangyo Kaisha, Ltd. (Kusatsu). As a control compound, SASP (Lot No. AA4383, Salazopyrin tablet $500 \mathrm{mg}$; Yoshitomi Pharmaceutical, Osaka), a drug generally used in the treatment of IBD, was used.

\section{Groups}

In addition to the 1,10 and $100 \mathrm{mg} / \mathrm{kg}$ per day groups of IS-741 for model rats, a SASP group in which the control compound SASP was administered at $100 \mathrm{~g} / \mathrm{kg}$ per day to model rats, a normal group in which distilled water used as vehicle was administered to normal rats, and a DSS

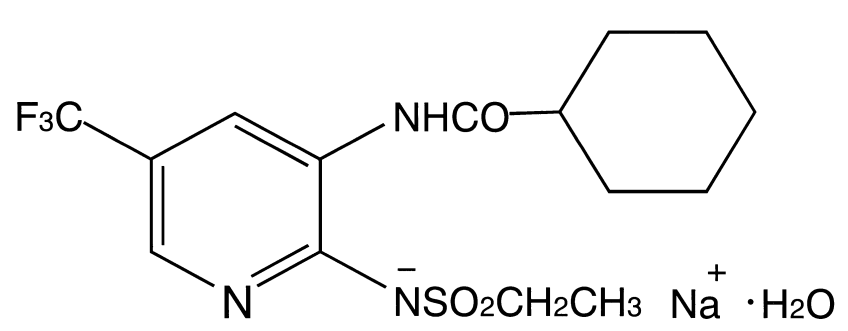

$\mathrm{C}_{15} \mathrm{H}_{19} \mathrm{~F}_{3} \mathrm{~N}_{3} \mathrm{NaO}_{3} \mathrm{~S} \cdot \mathrm{H}_{2} \mathrm{O}$

M.W. : 419.40

Fig. 1. Chemical structure of IS-741. (dextran sodium sulfate) group in which distilled water used as vehicle was administered to model rats were included. Each group included 10 animals.

\section{Preparation of model and administration of compounds}

IBD model animals were prepared by giving 3\% DSS (lot No. CKQ7309; Wako Pure Chemical Industries, Ltd., Osaka) in aqueous solution in drinking water to rats (Sprague Dawley, male, 7-week-old; Charles River Japan, Inc., Sagamihara) ad libitum for 11 days. Subsequently, 3\% DSS was changed to $1 \%$ DSS on the day of initiation of compound administration, and pathology was maintained during the administration period by similarly giving $1 \%$ DSS in drinking water. Purified water was similarly given to the animals in the normal group during the study period. Both the test compound and control compound were dissolved or suspended in distilled water, which was used as vehicle, and repeated oral administration of the respective compounds was conducted at $10 \mathrm{ml} / \mathrm{kg}$ once a day for 2 weeks. Vehicle alone was similarly administered to the animals in the normal group and the DSS group.

\section{Assessment}

Various tests were conducted in all the rats after completion of the administration period. Using a Coulter Counter Model T-660 automatic blood cell counter (Nikkaki Co., Ltd., Tokyo), white blood cell count (WBC), red blood cell count (RBC), hemoglobin $(\mathrm{Hb})$, hematocrit $(\mathrm{Ht})$ and platelets were determined. According to the method of Kimura et al. (28), samples stained with $1 \%$ Alcian blue were prepared, and the area of erosion in the large intestine was determined by an imaging analysis device ("Win ROOF" universal image processing device). To assess thickening of the intestinal wall, the length of the large intestine (colon and rectum) was determined at autopsy. Furthermore, histopathological samples from the large intestine were prepared by hematoxylin-eosin staining according to the usual method. The changes observed on histopathological examination were graded numerically (no change: 0 , slight change: 0.5 , mild change: 1.0 , moderate change: 2.0 , severe change: 3.0 ).

\section{Statistic assessment}

The results of blood tests, area of erosion in the large intestine, length of the large intestine and histopathological examination were statistically analyzed. Differences with a probability of $5 \%$ on both sides were considered significant $(P<0.05)$. In the case of blood test values, area of erosion in the large intestine and length of the large intestine, homoscedasticity was assessed by the $F$-test for comparison with the normal group and the DSS group and for comparison between the DSS group and SASP group. Student's $t$-test was applied in cases of homoscedasticity, and Aspin- 
Welch's $t$-test applied in cases of non-homoscedasticity. For comparison of the DSS group and the three IS-741dosing groups, uniformity $(P<0.01)$ was tested by the Bartlett method, and Williams' multiple comparison was performed in cases of uniformity. In cases of non-uniformity, log-transformation of data was performed for a further uniformity test $(P<0.05)$ by Bartlett's method. In cases of uniformity, the log-transformed data were examined by Williams' multiple comparison. Only the erosion area values were transformed into log values for analysis in the present study. For histopathological test results, Wilcoxon's rank-sum test was used for comparison between the normal group and DSS group and between the DSS group and SASP group, while Shirley-Williams' multiple comparison (separate ranking) was used for comparison between the DSS group and IS-741 groups. Values are expressed as the mean \pm S.D. of ten animals.

\section{RESULTS}

On hematological examination, a decrease in $\mathrm{RBC}, \mathrm{Hb}$ and $\mathrm{Ht}$ as well as an increase in WBC were noted in the DSS group compared with the normal group. Compared with the DSS group, the magnitude of decrease in RBC and $\mathrm{Hb}$ was less in all the IS-741 groups. Furthermore, the magnitude of decrease in Ht was also less in the IS-741 100 group. On the other hand, compared with the DSS group, the magnitude of decrease in $\mathrm{RBC}, \mathrm{Hb}$ and $\mathrm{Ht}$ was less in the SASP group (Fig. 2).

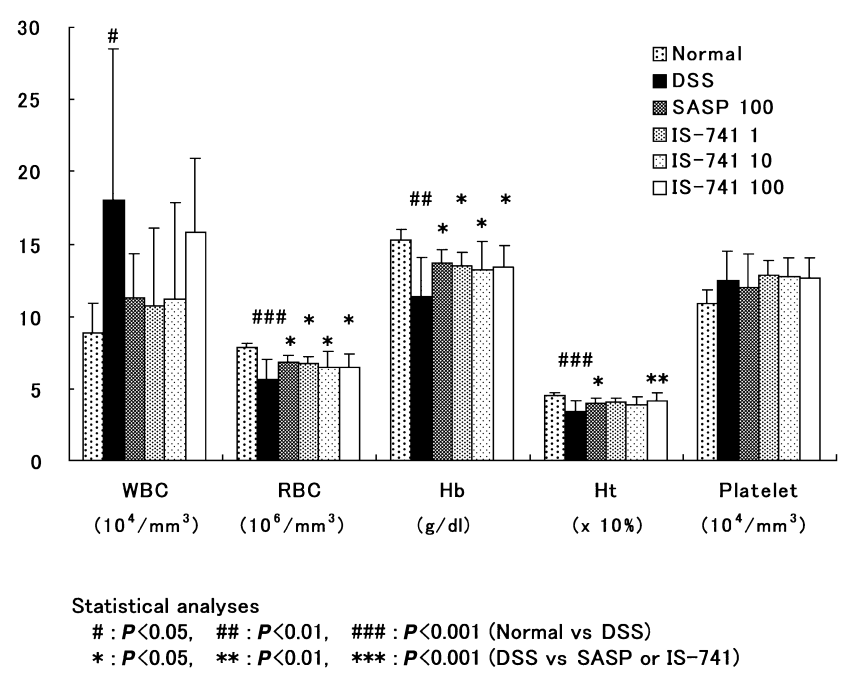

Fig. 2. Hematological examination. Compared with the normal group, WBC was increased $(P<0.05)$ and $\mathrm{RBC}, \mathrm{Hb}$ and $\mathrm{Ht}$ were decreased $(P<0.001, P<0.01$ and $P<0.001$, respectively) in the DSS group. The decreases in RBC and $\mathrm{Hb}$ were inhibited in the SASP group and all the IS-741 groups $(P<0.05$, all groups). Furthermore, the decrease in Ht was inhibited in the SASP group and IS-741 100 group $(P<0.05$ and $P<0.01$, respectively).
Erosion area was not detected in the normal group, but was high in the DSS group. Compared with the DSS group, erosion area was reduced in all the IS-741 groups and the SASP group (Fig. 3). The length of the large intestine was reduced in the DSS group compared with the normal group, reflecting thickening of the intestinal wall, a finding associated with erosion. Compared with the DSS group, less decrease in length of the large intestine was noted in all the IS-741 groups and the SASP group (Fig. 4).

On histopathological examination, sloughing of tissue from the mucosal epithelium to the lamina propria mucosae

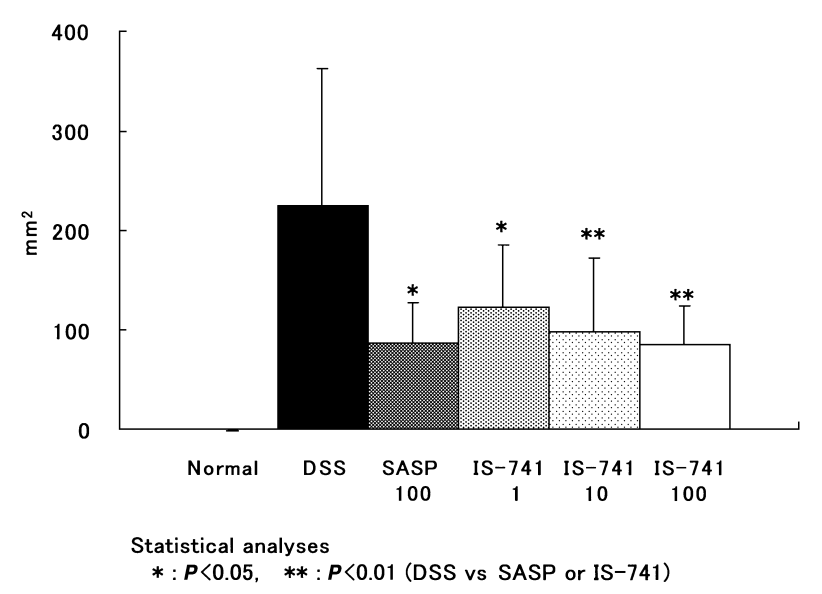

Fig. 3. Measurement of area of erosion in the large intestine. Compared with the normal group, the area of erosion was increased $(P<0.05)$ in the DSS group. Compared with the DSS group, the area of erosion was decreased in the SASP group and the 1, 10 and 100 groups of IS-741 $(P<0.05$ and $P<0.05, P<0.01$ and $P<0.01$, respectively).

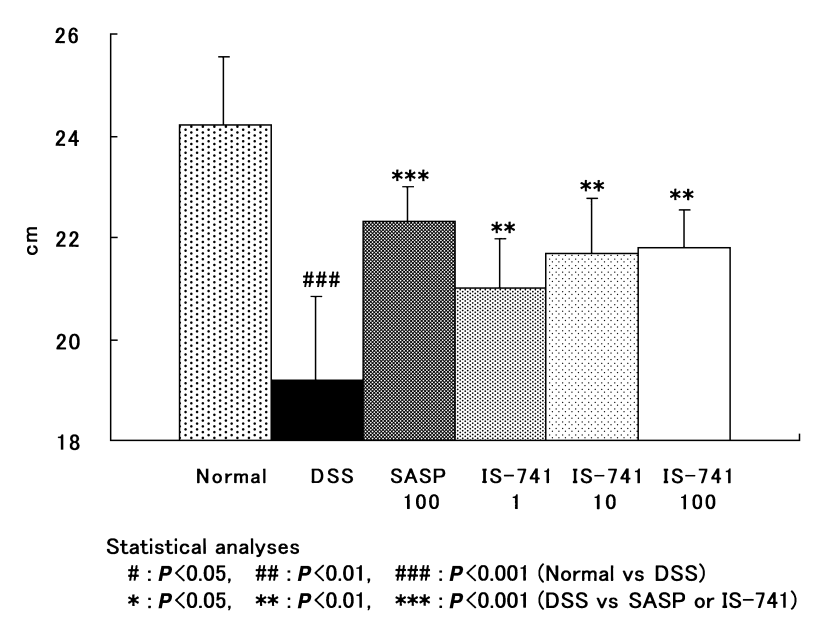

Fig. 4. Measurement of length of the large intestine. Compared with the normal group, a tendency toward decreased large intestine length was observed in the DSS group $(P<0.001)$. Compared with the DSS group, the extent of decrease in length was smaller in the SASP group and all the IS-741 groups $(P<0.001$ and $P<0.01$, respectively). 

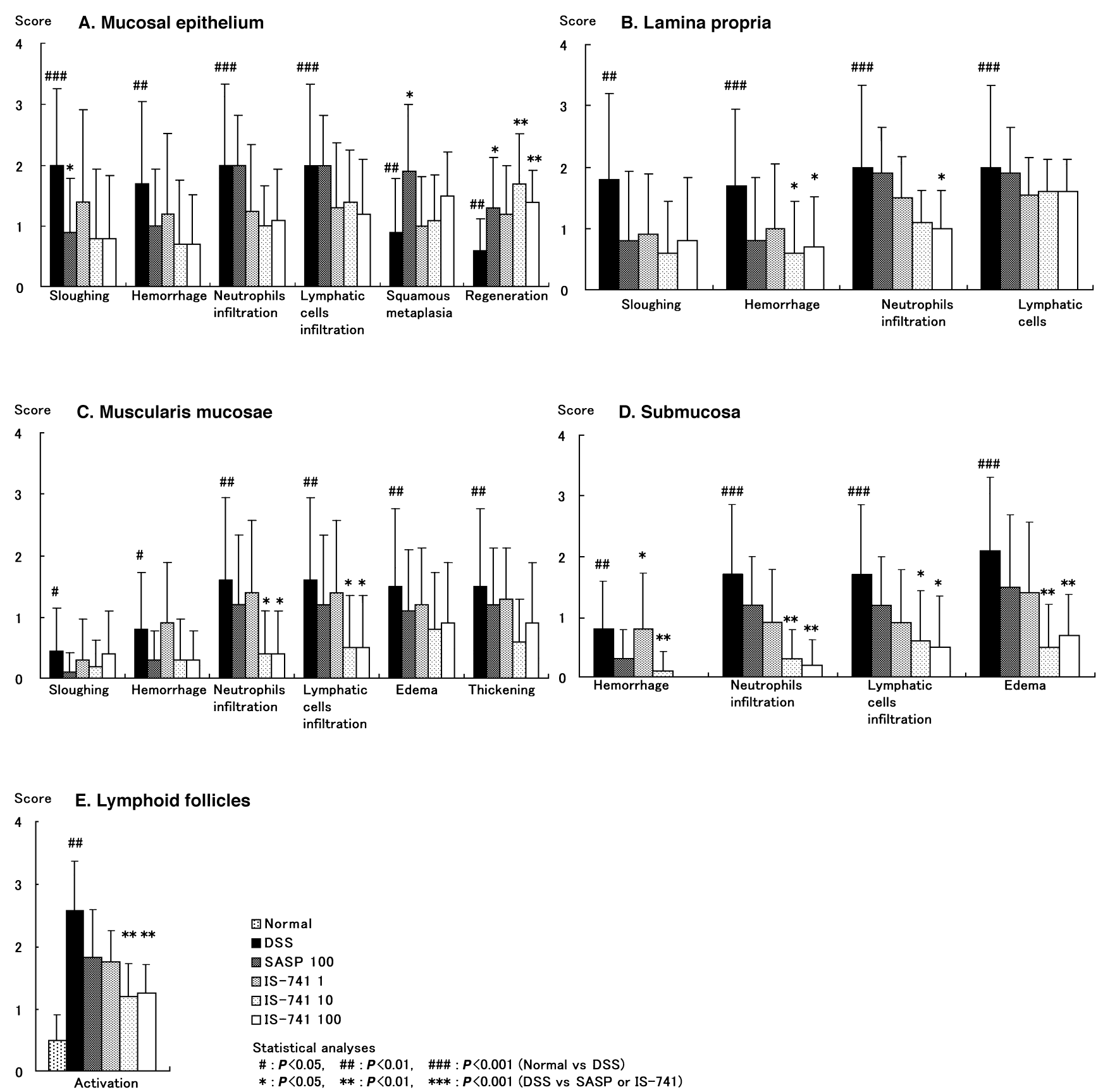

Fig. 5. Scores for histopathological changes in the mucosal epithelium (A), lamina propria (B), muscularis mucosae (C), submucosa (D) and lymphoid follicles (E) of the large intestine. A: Although sloughing $(P<0.001)$, hemorrhage $(P<0.01)$, neutrophil infiltration $(P<0.001)$, lymphatic cell infiltration $(P<0.001)$, and metaplasia and regeneration (both, $P<0.01)$ were observed in the DSS group, sloughing was decreased $(P<0.05)$, and metaplasia and regeneration were enhanced $(P<0.05)$ in the SASP group. Enhanced regeneration $(P<0.01)$ was noted in the IS-741 10 and 100 groups. B: Sloughing $(P<0.01)$, hemorrhage $(P<0.001)$, neutrophil infiltration $(P<0.001)$ and lymphatic cell infiltration $(P<0.001)$ were observed in the DSS group. These changes were not alleviated in the SASP group, but hemorrhage was decreased $(P<0.005)$ in the IS-741 10 and 100 groups, and neutrophil infiltration was decreased $(P<0.05)$ in the IS-741 100 group. C: Sloughing $(P<0.05)$, hemorrhage $(P<0.05)$, neutrophil infiltration $(P<0.01)$, lymphatic cell infiltration $(P<0.01)$, edema $(P<0.01)$ and thickening $(P<0.01)$ were observed in the DSS group. These changes were not alleviated in the SASP group, but neutrophil infiltration was decreased in the IS-741 10 and 100 groups $(P<0.01$, both groups). D: Hemorrhage $(P<0.05)$, neutrophil infilration $(P<0.01)$, lymphatic cell infiltration $(P<0.01)$ and edema $(P<0.01)$ were observed in the DSS group. These changes were not alleviated in the SASP group, while improvement was noted in the IS-741 10 and 100 groups in hemorrhage $(P<0.05, P<0.01$, respectively), neutrophil infiltration $(P<0.01$, both groups), lymphatic cell infiltration $(P<0.05$, both groups $)$ and edema $(P<0.01$, both groups). E: The activation of lymphoid follicles $(P<0.05)$ observed in the DSS group was not alleviated in the SASP group but was inhibited in the IS-741 10 and 100 groups. A - D: Any change was not observed in the normal group. 
A

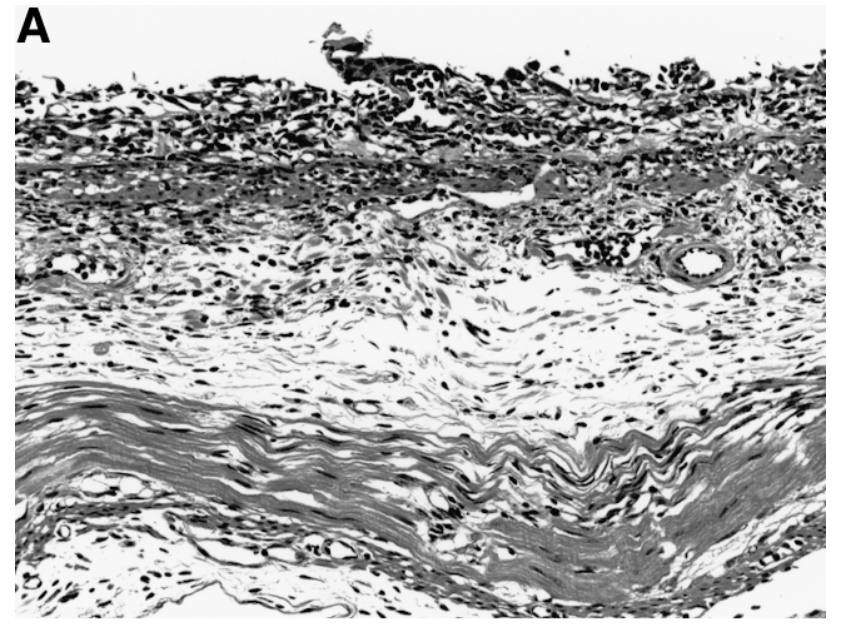

B

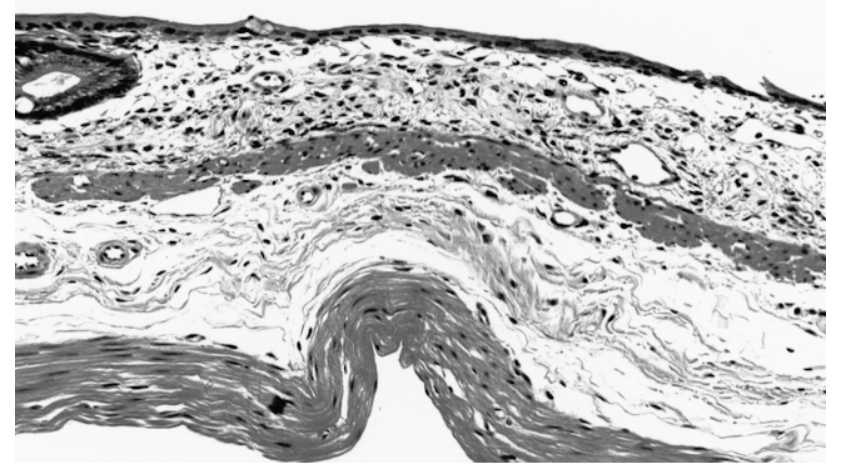

Fig. 6. Histopathological observations for the DSS group (A) and the IS-741 100 group (B) of the large intestine. Photo A for the DSS group reveals sloughing of tissue from the mucosal epithelium to the lamina propria and advanced neutrophil and lymphatic cell infiltration as well as edema from the lamina propria to submucosal tissue as major lesions. Photo B for the IS-741 100 group reveals regeneration and metaplasia, although slight, in the mucosal epithelium, and neutrophil and lymphatic cell infiltration as well as edema were markedly decreased from the lamina propria to submucosal tissue.

of the large intestine (sometimes reaching the muscularis mucosae), hemorrhage, and infiltration of neutrophils and lymphatic cells in sites of the large intestine from the mucosal epithelium to the submucosa were noted as major lesions in the DSS group and were not present in the normal group (Figs. 5 and 6).

In addition to these changes, edema originating from the muscularis mucosae to the submucosa, thickening of the muscularis mucosae and hyperplasia of lymphoid follicles in the submucosa were observed. Furthermore, regeneration or squamous metaplasia in the mucosal epithelium, though slight, was also observed (Figs. 5 and 6). Hyperplasia of lymphatic cells was noted in mesenteric lymph nodes. In the IS-741 10 group, the hemorrhage in the lamina propria layer and submucosa, neutrophil or lym- phatic cell infiltration in the muscularis mucosae and submucosa, edema in the submucosa and hyperplasia of lymphoid follicles were alleviated. Regeneration of the mucosal epithelium was also noted. In addition to the improvements observed in the IS-741 10 group, the neutrophil infiltration in the muscularis mucosae was alleviated in the IS-741 100 group. Especially noteworthy in the latter group was the decrease in neutrophil infiltration in the submucosa. Hemorrhage and edema in the same sites of the large intestine were also markedly alleviated. On the other hand, sloughing of the mucosal epithelium was significantly alleviated in the SASP group. Though squamous metaplasia or regeneration of the mucosal epithelium was noted, the former was predominant in the SASP group (Figs. 5 and 6).

\section{DISCUSSION}

The DSS-induced colitis model is reported to be useful for evaluation of therapeutic effects against ulcerative colitis, since erosion occurs frequently in the rectum with good reproducibility in it $(28-30)$. Increased erosion area was noted in the rectum in the DSS group in this study. Histopathological examination of erosion sites indicated sloughing of tissue from mucosal epithelium to the tunica propria, hemorrhage extending from mucosal epithelium to submucosa, marked inflammatory cell infiltration mainly with neutrophils and lymphocytes, and edema as the major lesions. The length of the large intestine including other than the sites of erosion, was decreased, reflecting thickening of the intestinal wall. Furthermore, hematological examination indicated a tendency toward anemia in addition to increased WBC. We speculate that the tendency toward anemia indicated melena due to hemorrhage of the rectum, based on results of histopathological examination. Based on the above findings, the DSS rat model used in this study is considered a valid model of ulcerative colitis for humans. IS-741 at 1,10 and $100 \mathrm{mg} / \mathrm{kg}$ per day alleviated mucosal erosion in the rectum and decreased length of the large intestine. It also alleviated the anemia assumed to be due to the melena caused by rectal erosions. The effect observed at each dose of IS-741 was nearly equivalent to that of SASP at $100 \mathrm{mg} / \mathrm{kg}$ per day.

It has already been reported that IS-741 inhibited leukocyte infiltration in a severe acute pancreatitis model, alleviates lesions of pancreatitis and multiple organ failure, and has a life-saving effect in an animal model of severe acute pancreatitis $(23-27)$. It has also been reported that the inhibition by IS-741 of leukocyte infiltration is based on inhibition of cell adhesion (22, 23). IS-741 acts on HL-60 (a human promyelocytic leukemia line) and inhibits the adhesion of HL-60 to vascular endothelial cells (22). Recent investigational results suggest that IS-741 inhibits cell 
adhesion by inhibiting cluster formation of LFA-1 (lymphocyte function associated antigen-1, CD11a/CD18) with activation of LFA-1 (H. Shikama et al., unpublished data).

Although no report of a clinically effective synthesized anti-inflammatory drug that acts on integrin is available, attempts have been made to inhibit the function of inflammation through integrin molecules by neutralizing antibody in various inflammation models, and the efficacy attained in these attempts has been reported $(31-36)$.

On the other hand, considerable involvement of the expression of adhesion molecules in the vascular endothelial cells in the pathology of IBDs has been reported in addition to that of abnormal cytokines produced by macrophages and lymphocytes in the tunica propria (13). The adhesion molecules ICAM-1 (intercellular adhesion molecule-1), VCAM-1 (vascular cell adhesion molecule-1) and E-selectin are expressed in vascular endothelial cells by cytokines including IL-1 and TNF in sites of inflammation. ICAM-1 accounts for the majority of adhesion molecules expressed in vascular endothelial cells in the periphery of erosion sites in patients with active-stage IBD (37). According to a study of human intestinal microvascular endothelial cells (HIMECs), ICAM-1 is consitutively expressed by such cells (38). On the other hand, LFA-1, Mac-1 (CD11b/CD18), VLA-4 (very late activation antigen) or other integrins are expressed in blood cells, including lymphocytes, neutrophils and macrophages, and play important roles in the migration of inflammatory cells to sites of inflammation. As noted above, ICAM-1 expressed in vascular endothelial cells and its ligand LFA-1 expressed in blood cells are thought to play important roles in inflammatory cell infiltration in ulcerative colitis as well.

Lymphocytes function throughout remission and the active stage of ulcerative colitis in the background. In addition to lymphocytes and plasma cells, infiltration by neutrophils, macrophages and eosinophils is observed in the mucosal layer of the large intestine during the active stage, yielding an acute-on-chronic picture. T-cell adhesion in human intestinal fibroblasts (HIF) derived from ulcerative colitis patients is higher than that in HIF from patients with other diseases (as demonstrated by binding assay with MOLT-4, a T-cell strain with LFA-1 on the surface). This phenomenon is inhibited by processing of anti-ICAM-1 antibody, and the LFA-1 and ICAM-1 adhesion pathway is also deeply involved in the interaction of T-cells and fibroblasts in the intestinal tract (39). In ulcerative colitis, fibroblasts retain T-cells that have wandered from vessels in the interstitium and contribute to persistent cellular activation.

The effects of IS-741 on LFA-1 function and its marked inhibition of infiltration by neutrophils and lymphocytes into tissues observed in this study suggest that IS-741 inhibits adhesion of leukocytes to vascular endothelial cells or intestinal tissues by inhibiting the function of integrin and thereby exhibits an anti-inflammatory effect. The above results suggested that it is possible to treat ulcerative colitis with a compound that inhibits cell adhesion.

Repeated oral administration of IS-741 at 1, 10 and $100 \mathrm{mg} / \mathrm{kg}$ per day for 2 weeks inhibited the leukocyte infiltration that plays an important role in IBD by inhibiting the infiltration of inflammatory cells via inhibition of cell adhesion, which is the mechanism of action of this compound, and consequently alleviated intestinal erosion and intestinal thickening, an associated lesion. In addition, IS-741 alleviated the anemia due to melena caused by erosion. These results were comparable to those obtained with SASP, the drug of first choice in clinical treatment, suggesting that IS-741 may be useful for the treatment of IBD.

\section{Acknowledgments}

The authors would like to extend their appreciation to Dr. S. Mizukoshi (Central Research Institute, Ishihara Sangyo Kaisha, Ltd.) who provided the opportunity to conduct this study, to Dr. I. Kimura (Environmental Biological Life Science Research Center, Inc.) who supervised this study, and to the staff who cooperated with this study.

\section{REFERENCES}

1 Zifroni A, Treves AJ, Sachar DB and Rachmilewitz D: Prostanoid synthesis by cultured intestinal epithelial and mononuclear cells in inflammatory bowel disease. Gut 24, 659-664 (1983)

2 Sharon P and Stenson WF: Enhanced synthesis of leukotriene $\mathrm{B}_{4}$ by colonic mucosa in inflammatory bowel disease. Gastroenterology 86, 453 - 460 (1984)

3 van Hogezand RA and Verspaget HW: New therapies for inflammatory bowel disease: an update on chimeric anti-TNF alpha antibodies and IL-10 therapy. Scand J Gastroenterol Suppl 223, 105 - 107 (1997)

4 Lang KA and Peppercprn MA: Promising new agents for the treatment of inflammatory bowel disorders. Drugs RD 1, 237 244 (1999)

5 Allison MC and Pounder RE: Cyclosporin for Crohn's disease. Aliment Pharmacol Ther 1, 39 - 43 (1987)

6 Shelly ED and Shelly WB: Cyclosporine therapy for pyoderma gangrenosum associated with sclerosing cholangitis and ulcerative colitis. J Am Acad Dermatol 18, 1084 - 1088 (1988)

7 Harris ML, Schiller HJ, Reilly PM, Donowitz M, Grisham MB and Bulkley GB: Free radicals and other reactive oxygen metabolites in inflammatory bowel disease: Acute consequence, or epiphenomenon? Pharmacol Ther 53, 375 - 408 (1992)

8 Middleton SJ, Shorthouse M and Hunter JO: Increased nitric oxide synthesis in ulcerative colitis. Lancet 341, 456-466 (1993)

9 Sawada K, Ohnishi K, Fukui S, Yamamura M, Amano K, Satomi $\mathrm{Ma}$ and Shimoyama T: Leukocytapheresis therapy with leukocyte removal filter for inflammatory bowel disease. J Gastroenterol 30, 124 - 127 (1995)

10 Sasaki M, Tsujikawa T, Fujiyama Y and Bamba T: Leukocyta- 
pheresis therapy for severe ulcerative colitis. Ther Apher 2, $101-104$ (1998)

11 Noguchi M, Hiwatashi N, Hayakawa T and Toyota T: Leukocyte removal filter-passed lymphocyte produce large amounts of interleukin-4 in immunotherapy for inflammatory bowel disease: role of bystander suppression. Ther Apher 2, 109 - 114 (1998)

12 Yajima T, Takaishi H, Kanai T, Iwao Y, Watanabe M, Ishii H and Hibi T: Predictive factors of response to leukocytapheresis therapy for ulcerative colitis. Ther Apher 2, 115 - 119 (1998)

13 Nakamura S, Ohtani H, Watanabe Y, Fukushima K, Matsumoto T, Kitano A, Kobayashi K and Nagura H: In situ expression of the cell adhesion molecules in inflammatory bowel disease. Evidence of immunologic activation of vascular endothelial cells. Lab Invest 69, 77 - 85 (1993)

14 Bernstein CN, Sargent M, Rawsthorne P and Rector E: Peripheral blood lymphocyte beta 2 integrin and ICAM expression in inflammatory bowel disease. Dig Dis Sci 42, 2338 - 2349 (1997)

15 Taniguchi T, Tsukada H, Nakamura H, Kodama M, Fukuda K, Saito T, Miyasaka M and Seino Y: Effects of the anti-ICAM-1 monoclonal antibody on dextran sodium sulphate-induced colitis in rats. J Gastroenterol Hepatol 13, 945 - 949 (1998)

16 Giacomelli R, Passacantando A, Frieri G, Parzanese I, D'Alo S, Vernia P, Pimpo MT, Petrucci C, Caprilli R, Cifone MG and Tonietti G: Circulating soluble factor-inhibiting natural killer (NK) activity of fresh peripheral blood mononuclear cells (PBMC) from inflammatory bowel disease (IBD) patients. Clin Exp Immunol 115, 72 - 77 (1999)

17 Woywodt A, Ludwig D, Neustock P, Kruse A, Schwarting K, Jantschek G, Kirchner H and Stange EF: Mucosal cytokine expression, cellular markers and adhesion molecule in inflammatory bowel disease. Eur J Gastrol Hepatol 11, 267-276 (1999)

18 Meenan J, Hommes DW, Mevissen M, Dijkhuizen S, Soule H, Moyle M, Buller HR, ten Kate FW, Tytgat GN and van Deventer SJ: Attenuation of the inflammatory response in an animal colitis model by neutrophil inhibitory factor, a novel beta 2-integrin antagonist. Scand J Gastroenterol 31, 786 - 791 (1996)

19 Yacyshyn BR, Bowen-Yacyshyn MB, Jewell L, Tami JA, Bennett CF, Kisner DL and Schanahan WR Jr: A placebocontrolled trial of ICAM-1 antisense oligonucleotide in the treatment of Crohn's disease. Gastroenterology 114, $1133-1142$ (1998)

20 Nakajima H, Munakata A and Yoshida Y: Adverse effects of sulfasalazine and treatment of ulcerative colitis with mesalazine. J Gastroenterol 30, Suppl 8, 115 - 117 (1995)

21 Stein RB and Hnauer SB: Comparative tolerability of treatment for inflammatory bowel disease. Drug Saf 23, 429 - 448 (2000)

22 Shikama H, Yotsuya S, Satake S, Sugi H and Kato M: Effect of IS-741 on cell adhesion between human umbilical vein endothelial cells and HL-60 cells. Biol Pharm Bull 22, 127-131 (1999)

23 Yotsuya S, Shikama H, Nakano I, Sakai K, Kato M, Sugi H, Takada $\mathrm{H}$ and Koga Y: A novel synthetic anti-acute pancreatitis agent, IS-741. Digestion 60, Suppl 1, 34 - 39 (1999)

24 Isaji S, Hayashi J, Higashiguchi T, Yokoi H, Ogura Y, Noguchi $\mathrm{T}$ and Kawarada Y: Effect of IS-741 (a new synthetic antiinflammatory agent) on acute necrotizing pancreatitis in dogs. Digestion 60, Suppl 1, $47-51$ (1999)

25 Yamaguchi Y, Okabe K, Liang J, Matsumura F, Akizuki E, Matsuda T, Ohshiro H, Nakano S, Ishihara K and Ogawa M:
The novel carboxamide derivative IS-741 reduces neutrophil chemoattractant production by bronchoalveolar macrophages in rats with cerulein-induced pancreatitis complicated by sepsis. Digestion 60, Suppl 1, $52-56$ (1999)

26 Liang J, Yamaguchi Y, Matsumura F, Okabe K, Akizuki E, Ohshiro H, Nakano S, Ishihara K and Ogawa M: Novel carboxamide derivative (IS-741) attenuates lung injury in rats with cerulein-induced pancreatitis complicated by endotoxemia. Dig Dis Sci 44, 341 - 349 (1999)

27 Yamauchi J, Sunamura M, Shibuya K, Takeda K, Kobari M and Matsuno S: A novel diamino-pyridine derivative prevents excessive leukocyte infiltration in aggravation of acute necrotizing pancreatitis. Digestion 60, Suppl 1, 40 - 46 (1999)

28 Kimura I, Kawasaki M, Nagahama S, Matsuda A, Kataoka M and Kokuba Y: Determination of the acute moiety of BX661A, a new therapeutic agent for ulcerative colitis, by studying its therapeutic effect on ulcerative colitis induced by dextran sulfate sodium in rats. Arzneimittelforschung 48, 1091 - 1096 (1998)

29 Hori Y, Hoshino J, Yamazaki C, Sekiguchi T, Miyauchi S, Mizuno S and Horie K: Effect of lecithinized-superoxide dismutase on the rat colitis model induced by dextran sulfate sodium. Jpn J Pharmacol 74, 99 - 103 (1997)

30 Kitajima S, Takuma S and Morimoto M: Histological analysis of murine colitis induced by dextran sulfate sodium of different molecular weight. Exp Anim 49, 9 - 15 (2000)

31 Issekutz AC and Issekutz TB: Monocyte migration to arthritis in the rat utilizes both $\mathrm{CD} 11 / \mathrm{CD} 18$ and very late activation antigen 4 integrin mechanisms. J Exp Med 181, 1197-1203 (1995)

32 Burkly LC, Jakubowski A and Hattori M: Protection against adoptive transfer of autoimmune diabetes mediated through very late antigen 4 (VLA-4) integrin. Diabetes 43, 529 - 534 (1994)

33 Rabb HA, Olivenstein R, Issekutz TB, Renzi PM and Martin JG: The role of the leukocyte adhesion molecles VLA-4, LFA-1 and Mac-1 in allergic airway responses in the rat. Am J Respir Crit Care Med 149, 1186 - 1191 (1994)

34 Issekutz TB: Dual inhibition of VLA-4 and LFA-1 maximally inhibits cutaneous delayed-type hypersensitivity-induced inflammation. Am J Pathol 143, 1286 - 1293 (1993)

35 Kondo S, Kono T, Brown WR, Pastore S, McKenzie RC and Sauder DN: Lymphocyte function-associated antigen-1 is required for maximum elicitation of allergic contact dermatitis. Br J Dermatol 131, 354 - 359 (1994)

36 Rosenbaum JT and Boney RS: Efficacy of antibodies to adhesion molecules, CD11a or CD18, in rabbit models of uveitis. Curr Eye Res 12, 827 - 831 (1993)

37 Morise K, Yamaguchi T, Kuroiwa A, Kanayama K, Matsuura T, Shinoda M, Yamamoto H, Horiuchi Y, Furusawa A, Iwase H, Kusugami $\mathrm{K}$ and Nagura H: Expression of adhesion molecules and HLA-DR by macrophages and dendritic cells in aphthoid lesions of Crohn's disease: an immunocytochemical study. J Gastroenterol 29, 257 - 264 (1994)

38 Binion DG, West GA, Ina K, Ziats NP, Emancipator SN and Fiocchi C: Enhanced leukocyte binding by intestinal microvascular endothelial cells in inflammatory bowel disease. Gastroenterology 112, 1895 - 1907 (1997)

39 Ina K, Kusugami $\mathrm{K}$ and Fiocchi $\mathrm{C}$ : Enhanced interaction of intestinal fibroblasts with T-cells in inflammatory bowel disease. Gastroenterology 110, A930 (1996) 